\title{
Immobilized artificial membrane chromatography: from medicinal chemistry to environmental sciences
}

\author{
Fotios Tsopelas ${ }^{1 *}$, Chrysanthos Stergiopoulos ${ }^{1}$ and Anna Tsantili-Kakoulidou ${ }^{2}$ \\ ${ }^{1}$ Laboratory of Inorganic and Analytical Chemistry, School of Chemical Engineering, National Technical University of \\ Athens, Iroon Polytechniou 9, 15780 Athens, Greece. \\ ${ }^{2}$ Laboratory of Pharmaceutical Chemistry, School of Pharmacy, University of Athens, Panepistimiopolis, Zografou, 157 \\ 71 Athens, Greece.
}

*Corresponding Author: E-mail: ftsop@central.ntua.gr Phone: +30 $2107724022,+302107723210$

Received: May 21, 2018; Revised: August 15, 2018; Available online: September 22, 2018

\begin{abstract}
Immobilized Artificial Membrane (IAM) chromatography constitutes a valuable tool for medicinal chemists to prioritize drug candidates in early drug development. The retention on IAM stationary phases encodes lipophilicity, electrostatic and other secondary interactions contrary to traditional octanol-water partitioning. An increasing number of publications in recent years have suggested that IAM indices, including isocratic log $k_{(I A M)}$ or extrapolated log $k_{w(I M)}$ retention factors, chromatographic hydrophobicity index $\mathrm{CH}_{(\text {IAM) }}$ or the polarity parameter $\Delta \log k_{w(I A M)}$ can successfully model the passage of xeniobiotics through biological membranes and barriers and predict pharmacokinetic properties, often in combination with additional descriptors. Examples referring to the modeling of human oral absorption, blood-brain penetration and skin partition are described. More recently, IAM chromatography has been applied to estimate toxicological endpoints in regard to drug safety, such as phospholipidosis potential, or in regard to chemical risk hazards including the bioconcentration factor and aquatic organisms' toxicity. The promising results in both medicinal chemistry and in environmental science in combination with the speed, reproducibility and low analyte consumption suggest that a broader application of IAM chromatography in the early drug discovery process and in ecotoxicity may save time and money in initial drug candidate selection and will contribute to a reduced risk hazard of chemicals.
\end{abstract}

\section{Keywords}

IAM chromatography; lipophilicity; phospholipophilicity; membrane permeability; human oral absorption; ecotoxicity; aquatic toxicity; bioconcentration factor (BCF)

\section{Introduction to IAM Chromatography}

High-performance liquid chromatography (HPLC) is a powerful analytical technique based on the differentiation in the elution of different compounds in a given sample when passed through a chromatographic column (stationary phase) by the flow of a mobile phase. Thus, dissolved in the mobile phase chemicals participate in a dynamic equilibrium between the mobile and stationary phase and their elution expresses their distribution coefficients between the two phases, depending on various primary and secondary interactions. As several pharmacokinetic and ecotoxicological properties involve a dynamic distribution of xenobiotics between general circulation and tissues, or aqueous environment and tissues of 
aquatic organisms, HPLC can be used in order to predict pharmacokinetic properties and environmental risk indices. The strategy and the key advantages of using HPLC as a tool to achieve biomimetic conditions have been addressed by Valko [1,2].

The use of a chromatographic index (e.g. retention factor) as a measure of a biological endpoint (e.g. pharmacokinetic properties, toxicity and ecotoxicological profile) has generated quantitative retentionactivity relationships (QRAR) [3]. The main advantage of QRAR is that it is not necessary to know the values of the molecular descriptors of the compound under investigation in order to estimate its pharmacokinetic or ecotoxicological profile as in classic quantitative structure-activity relationship (QSAR) studies. This is important in the case of new compounds.

The first attempt of introducing HPLC to the modelling of biological processes with the employment of reversed-phase liquid chromatography as an alternative for lipophilicity assessment was made in order to overcome certain disadvantages of direct partitioning experiments [4]. Lipophilicity was known as a predominant determinant in drug transport across membranes as proved by the traditional Hansch analysis [5]. The development of biomimetic chromatographic columns provided a substantial advance for the experimental evaluation of ADMET properties in the early stages of drug/chemical development, combining the simulation of biological processes with rapid measurements [6-8]. In pharmaceutical and environmental sciences three types of biomimetic chromatography are used; immobilized artificial membrane (IAM), immobilized plasma protein chromatography as well as biopartitioning micellar chromatography (BMC). The first two types are based on biomimetic stationary phases, IAM and immobilized plasma protein (i.e. human serum albumin and Alpha-1 acid glycoprotein), respectively. The third uses reversed phase columns and the biological environment is mimicked by micellar mobile phases obtained upon addition of a surfactant above its critical micellar concentration $[9,10]$. The retention under biomimetic conditions is referred to as biomimetic properties which may play an essential role in the drug design process of selecting the appropriate compound with the right pharmacokinetic/toxicological profile to become an effective drug [11]. Biomimetic chromatography has the advantage of combining simulation of the biological environment with high speed, is economic and user friendly and requires only a small amount of the analyte without the necessity to be pure $[12,13]$.

Of particular importance is the modelling of biological membranes which constitute barriers, which bioactive compounds have to cross in order to reach their molecular target and exert their pharmacological actions or their potential adverse effects. Membrane permeability can be modeled by a range of approaches, starting with the traditional octanol-water partitioning, or liposomes partitioning and proceeding to the development of different cell cultures or artificial membrane assays, IAM chromatography however, constitutes a powerful alternative due to its superior performance in terms of the simplicity of the experiments, high throughput, reproducibility and the possibility of automation. In this present review, the latest advances in IAM chromatography in both the pharmaceutical and the environmental sciences are presented and discussed.

\section{Types of IAM stationary phases and their interrelation}

IAM stationary phases are prepared by immobilization of phospholipids (e.g. phosphatidylcholine) on propylamino-silica skeleton. At present, three types of IAM columns are commercially available; the single chain IAM.PC (PC stands for "phosphatidylcholine"); the double-chain IAM.PC.MG (MG stands for "methylglycolate") and the IAM.PC.DD2 (DD stands for "drug discovery"). According to its producer IAM.PC and IAM.PC.MG are suggested for "membrane protein purification", while IAM.PC.DD2 is recommended 
for drug discovery purposes. IAM.PC.DD2 is available in 3, 10 and $15 \mathrm{~cm}$ long columns, IAM.PC in 15 and 30 $\mathrm{cm}$ columns, while IAM.PC.MG is produced in 3 and $15 \mathrm{~cm}$ length columns. All types of the columns have an i.d. of $4.6 \mathrm{~mm}$ and a stationary phase of $10 \mu \mathrm{m}$ particle size. An IAM fast- screen mini column ( $1 \mathrm{~cm} \mathrm{x}$ $3 \mathrm{~mm}$ ) is also available for rapid estimations of drug permeability in high- throughput screening programs for the characterization of large libraries of compounds [14].

According to the literature, not only the IAM.PC.DD2 column but also the IAM.PC.MG column are widely employed in pharmaceutical [15-20] and environmental research [21]. The difference between the two columns is in the end-capping of the free (unreacted) propylamino residues, performed either by methylglycolate or decyl/propyl anhydride as described in detail in the literature [6,7,22-24]. Previous comparative investigations on the two IAM stationary phases using a structurally diverse set of compounds $[16,18,25-27]$ showed a similar underlying elution mechanism with an almost 1:1 correlation between the retention factors measured in a pure aqueous phase, $\log k_{\mathrm{w}(\mathrm{IAM})}$. A representative interrelationship based on 161 structurally diverse compounds is expressed by Eq.(1) [27]:

$$
\begin{aligned}
& \log k_{\mathrm{w}(\text { IAM.DD2) }}=0.1437+1.0757 \cdot \log k_{\mathrm{w}(\text { IAM.PC.MG })} \\
& \left(N=161, R^{2}=0.93, s=0.314\right)
\end{aligned}
$$

Where $N$ is the number of compounds, $R^{2}$ is the correlation coefficient and $s$ is the standard deviation.

The similarity between the two columns permits the combination of retention factors measured on both IAM stationary phases in order to model biological processes.

\section{IAM indices and conditions used}

The most common approach for the employment of IAM chromatography is the simple determination of the retention factor of the solute, according to Eq. (2):

$$
k_{(I A M)}=\frac{t_{r}-t_{0}}{t_{0}}
$$

where $t_{\mathrm{r}}$ is the retention of the test compound and $t_{0}$ is the elution time of an unretained compound, such as sodium citrate, L- cysteine or ammonium oxalate [28]. The IAM retention factor, $k_{(\text {IAM) }}$, is proportional to the partition coefficient, $K_{(\mathrm{IAM})}$, between the IAM stationary phase and the mobile phase according to equation (3):

$$
k_{(\mathrm{IAM})}=K_{(\mathrm{IAM})} \times \frac{v_{\mathrm{s}}}{V_{\mathrm{m}}}
$$

where $V_{\mathrm{s}}$ and $V_{\mathrm{m}}$ are the volumes of stationary and mobile phase, respectively, whose ratio for a given column is constant. For IAM.PC.DD2, $V_{s} / V_{m}$ is equal to 0.053 [29] and, therefore, the $k_{(\text {IAM) }} / K_{(I A M)}$ relation is given according to Eq. (4):

$$
K_{(\mathrm{IAM})}=\frac{1}{0.053} \times k_{(\mathrm{IAM})}=18.9 \times \frac{t_{\mathrm{r}}-t_{0}}{t_{0}}
$$

In modelling biological processes or ecotoxicity, retention factors are usually used in their logarithmic form in order to achieve linear relationships with free energy. IAM measurements are performed at ambient temperature $[17,20,26,30-34]$ contrary to biopartitioning micellar chromatography which is usually employed at $36.5-37.0^{\circ} \mathrm{C}[9,10]$. Mobile phases consist of a buffer, typically phosphate-buffered saline (PBS) in order to mimic physiological conditions. A limitation in the preparation of mobile phases is 
their $\mathrm{pH}$ value, which should be adjusted to between 2.5 and 7.4. Although it is common to add an organic modifier to the mobile phases, IAM stationary phases can also be used with a pure aqueous phase $[26,28,31,34,35]$. In such cases, the measured log $k_{(\text {IAM) }}$ values correspond to the actual log $k_{\mathrm{w}(\mathrm{IAM})}$ values, where the symbol " $w$ " denotes the pure aqueous phase. In the case of compounds exhibiting strong affinity with the IAM stationary phase, acetonitrile can be added up to concentrations of $30 \%$ and $\log k_{\mathrm{w}(\mathrm{IAM})}$ values can be obtained by linear extrapolation of isocratic $\log k_{(\mathrm{IAM})}$ values, measured in the presence of at least four different acetonitrile fractions $(\varphi)$ in the mobile phase, according to Eq.(5):

$$
\log k_{(\mathrm{IAM})}=\log k_{\mathrm{w}(\mathrm{IAM})}-S \times \varphi
$$

where $S$ is the slope of the $\log k$ vs $\varphi$ relationship, calculated by linear regression. Comparative studies between actual and extrapolated $\log k_{\mathrm{w}(\mathrm{IAM})}$ values showed only small differences $[17,26]$. Such findings permit the combined use of actual $\log k_{\mathrm{w}(\mathrm{IAM})}$ values with extrapolated values in the case of lipophilic compounds. However, the use of mobile phases with high concentration of acetonitrile should be avoided as the structure of water layer on the stationary phase surface can be disrupted [36]. The use of methanol as an organic modifier should also be avoided as it can cause instability of the stationary phase due to methanolysis of the phospholipids $[37,38]$. To this point, the high inter- and intra-laboratory reproducibility of actual or extrapolated log $k_{\mathrm{w}(\mathrm{IAM})}$ values under standard conditions (buffer PBS and acetonitrile as organic modifier) should be highlighted [7].

As an alternative to using IAM retention factors, Valko has proposed the chromatographic hydrophobicity index for IAM chromatography, $\mathrm{CHI}_{(\mathrm{IAM})}[1,39,40]$, which, in recent literature, has been also termed as "membrane binding index" (IAM MB) [41]. $\mathrm{CHI}_{(\mathrm{IAM})}$ corresponds to the percentage of acetonitrile required for the equal partitioning of the solute between the mobile and the stationary phase (e.i. $\log k=0)$, which according to Eq.(5) is equal to $\varphi_{0}=\log k_{\mathrm{w}(\mathrm{IAM})} / \mathrm{S}$. The rapid determination of $\mathrm{CH}_{(\mathrm{IAM})}[1,39]$ can be easily performed by the measurement of the gradient elution times $\left(t_{\mathrm{g}}\right)$ measured under the appropriate gradient conditions. The obtained $t_{\mathrm{g}}$ for each analyte is converted to its $\mathrm{CHI}_{(\mathrm{IAM})}$ value by using a linear calibration equation regression line $\mathrm{CHI}_{(\mathrm{IAM})}=f\left(t_{\mathrm{g}}\right)$ obtained by using 9 standard compounds, namely octanophenone, heptanophenone, hexanophenone, valerophenone, butyrophenone, propiophenone, acetophenone, acetanilide and paracetamol [39]. In such experiments usually, ammonium acetate is the buffer, which is compatible with mass spectrometry, while acetonitrile is the organic modifier [39].

$\mathrm{CHI}_{(\mathrm{IAM})}$ can be further converted to log $k_{\mathrm{w}(\mathrm{IAM})}$ for the neutral form of the molecule by means of Eq.(6) which was established from 86 diverse drug molecules [11].

$$
\begin{aligned}
& \log k_{\mathrm{w}(\mathrm{IAM})}=0.046 \mathrm{CHI}_{(\mathrm{AMM})}+0.42 \\
& \left(n=86, R^{2}=0.94, s=0.29\right)
\end{aligned}
$$

\section{Relationship of IAM retention with lipophilicity and other molecular factors}

In order to unravel the potential applications of IAM chromatography in pharmaceutical and environmental sciences, it is crucial to investigate the elution mechanism, governing the IAM retention mechanism. There is general agreement that IAM retention is governed by lipophilicity, involving hydrophobicity, polarity and the additional factors, of electrostatic interactions in the case of ionizable compounds (e.g. deprotonated acids, protonated bases and zwitterions) with the charged centres of the phospholipids and the consequent formation of ionic bonds [17,21,26,31,34,38]. In such cases, IAM retention may be considered as a border case between passive diffusion and binding as was highlighted by Van Balen et al. [42]. The specific contribution of each parameter to the so-called "phospholipophilicity" 
can be expressed by Eq. (7) and has been discussed in a previous review $[7,8]$ :

Phospholipophilicity $=$ Hydrophobicity \pm Polarity + Electrostatic interactions

The sign of polarity in Eq. (7) can be negative reducing phospholipophilicity in an analogous manner to its effect on lipophilicity, or positive as a result of hydrogen bonding to the glycerol ester group of the phospholipids. Electrostatic interactions lead overall to enhanced retention, although repulsive forces between solute anions and IAM phosphate groups may also occur.

The crucial role of hydrophobicity to IAM retention can be highlighted by considering $\log k_{\mathrm{w} \text { (IAM) }} / \log P$ relationships, which are generally characterized by good statistics. In the case of ionizable compounds $\log k_{\mathrm{w}(\mathrm{IAM})}$ may still correlate better with $\log P$ than $\log D$ as a result of the partial compensation of ionization via electrostatic interactions [26]. However, the relationship of $\log k_{\mathrm{w}(\mathrm{IAM})}$ with $\log D$ can be considerably improved by introducing the positively $\left(\mathrm{F}^{+}\right)$or negatively charged $\left(\mathrm{F}^{-}\right)$molecular fraction of the analytes $[17,21,26,31,34]$ as additional parameters. Positive signs (higher for $\mathrm{F}^{+}$than for $\mathrm{F}^{-}$) are generated, as shown in Eq. (8) at $\mathrm{pH}=7.4$, supporting the overall positive contribution of electrostatic interactions.

$$
\begin{aligned}
& \log k_{\mathrm{w}(\mathrm{IAM})}=0.62 \times \log D_{7.4}+1.14 \times \mathrm{F}^{+}+0.48 \times \mathrm{F}^{-}+0.32 \\
& \left(\mathrm{n}=56, \mathrm{R}^{2}=0.938, \mathrm{~s}=0.413\right)
\end{aligned}
$$

The roustness of Equation (8) has been sucessfully validated by other sets of structurally diverse drugs $[17,26]$.

Other authors have confirmed the electrostatic interactions in IAM retention using linear free energy relationships (LFER) upon introduction of the ionization correction factors ( $\delta$-correction or $\mathrm{J}^{+}$and $\mathrm{J}^{-}$for ionic species) as additional parameters $[43,44]$. A representative equation obtained at $\mathrm{pH}=7.0$ is shown in Eq. (9) [44]:

$$
\begin{aligned}
& \log k_{\mathrm{w}(\mathrm{AM})}=-0.607+0.881 \cdot \mathrm{E}-0.562 \cdot \mathrm{S}-0.348 \cdot \mathrm{A}-1.794 \cdot \mathrm{B}+2.250 \cdot \mathrm{V}-0.245 \cdot \mathrm{J}^{+}+2.010 \cdot \mathrm{J} \\
& \left(n=68, R^{2}=0.896, \mathrm{~s}=0.431\right)
\end{aligned}
$$

In Eq. (9), the solute descriptors are as follows: $V$ is the McGowan's characteristic volume, $E$ is the excess molar refraction, $\mathrm{S}$ is the dipolarity/polarizability, and $\mathrm{A}$ and $\mathrm{B}$ are the hydrogen-bond acidity and basicity, respectively.

The additional terms, $\delta$-correction or $\mathrm{J}^{+}$and $\mathrm{J}^{-}$have positive signs (higher for cations than for anions). A possible explanation for the stronger electrostatic interactions of cations is the location of the IAM phosphate groups close to the hydrophobic part of phospholipids, in contrast to the choline nitrogen which is more exposed to the solvent effect at the outer terminal of the IAM surface $[21,26]$. Thus, phosphate anions enhance the partitioning of cations into the IAM stationary phase by attraction forces, while as already commented, repulsive forces may occur with anions [21,26]. However, in the case of very hydrophilic anions, partitioning into the hydrophobic core may not be the determinant factor and attraction by the positively charged choline nitrogen becomes similarly important. In such cases, retention is mainly governed by electrostatic interactions [17].

Comparative block relevance analysis of PLS models based on Volsurf descriptors further supports the similar information content of $\log k_{\mathrm{w}(\mathrm{IAM})}$ and $\log P$ for neutral compounds. As for bases and acids the analysis of $\log k_{\mathrm{w}(\mathrm{IAM})}$ (and also of $\log P$ ) generates a positive sign for the block of $\mathrm{H}$-bond donor (HBD) properties in accordance with the enhanced affinity to the IAM stationary phase, favoured for cations over anions, while the opposite sign (negative) is observed in the $\log D$ model [45]. 
The contribution of hydrogen bonding has also been reported [7,25,31,34] and Barbato [46] has discussed the role of glycerol as a hydrogen acceptor in solute retention. This may be the reason for only the moderate interrelation between $\log k_{\mathrm{w}(\mathrm{IAM})}$ on the two columns, IAM.PC.DD2 and IAM.PC.MG obtained for a set of 41 flavonoids, mainly as a result of their different content in alcoholic hydroxyl groups [20].

The polar/electrostatic interactions can be expressed as $\left(\Delta \log k_{\mathrm{w}(\mathrm{IAM})}\right)$, which is the difference between the experimental $\log k_{\mathrm{w}(\mathrm{IAM})}$ value and the $\log k_{\mathrm{w}(\mathrm{IAM})}$ estimated by a calibration equation of $\log k_{\mathrm{w}(\mathrm{IAM})}$ versus $\log P$, which is obtained for neutral compounds, interacting with the IAM stationary phase by a purely hydrophobic mechanism. $\Delta \log k_{\mathrm{w}(\mathrm{IAM})}$ is higher for poorly lipophilic compounds, while high lipophilicity produces negative values $[16,18,19,25]$.

As confirmed also by block relevance analysis at least for neutral compounds, $\Delta \log k_{\mathrm{w}(\mathrm{IAM})}$ is considered as a polarity index, which can be used in QSPR studies replacing other polarity parameters such as polar surface area (PSA) or $\Delta \log P_{\text {oct-alk }}[45]$.

Recently, the contribution of secondary interactions due to residual silanol and propylamine groups to the elution mechanism on IAM stationary phases has been investigated. Such secondary interactions are attributed to the change of the net charge of the IAM surface as a function of $\mathrm{pH}$. At $\mathrm{pH}>5$ there is an overall negative charge on the IAM surface due to the predominance of ionized silanol groups, while at lower $\mathrm{pH}$ protonated propylamine groups prevail resulting in a positive charge. Hence, at physiological conditions, an increased retention capacity of protonated bases can be anticipated and therefore, an overestimation of the affinity of such compounds to phospholipids. The resulting electrostatic effect is more pronounced in low salinities of eluents [47]. The role of silanophilic interactions in IAM retention has also been reported by other authors $[30,34,38]$.

The identification of the molecular factors expressed in IAM retention has provided tools for in silico prediction of phospholipophilicity. Ledbetter et al. [48] developed an algorithm for the prediction of $\log k_{\mathrm{w}(\mathrm{AM})}$ values of compounds based on fragments and correction factors using a data set which consisted of 22 aliphatic and 42 aromatic compounds. Recently, Russo et al. [27] proposed a model for prediction of $\log k_{\mathrm{w}(\mathrm{IAM})}$ values, involving four in silico calculated parameters; lipophilicity, hydrophilic/ lipophilic balance, molecular size and molecular flexibility. The work was based on their measurements on 205 experimental values made by them on IAM.PC.DD2 and IAM.PC.MG column types, to minimize inter-laboratory variability, leading to a good degree of accuracy $\left(R^{2}=0.85\right)$.

\section{The applications of IAM chromatography to predict ADMET properties in pharmaceutical sciences}

Interactions between drugs and immobilized phospholipids, expressed in IAM retention, can serve as important indices for various processes, such as drug permeability and absorption (considering passive diffusion as the main process), tissue and subcellular distribution as well as toxicity due to functional and morphological changes in cells as for instance phospholipidosis. Comparative studies have shown that IAM retention factors correlate with Caco-2 permeability [49-51] or with permeability through MDCK cell lines, two standard protocols which used to measure permeability in vitro [17].

Of significant importance are the applications of IAM chromatography to model gastrointestinal absorption/ bioavailability. It is known that passive permeation routes for drugs involve diffusion through the aqueous pores at the tight junctions, the so-called paracellular absorption and transcellular absorption through the enterocyte itself [52]. Published investigations have reported mainly the establishment of nonlinear models for transcellular based human oral absorption (HOA) combining IAM retention with other 
physicochemical properties, such as molecular weight (MW) or polarity terms. Yoon et al. reported a sigmoidal model for a data set of 40 structurally diverse drugs [53] using isocratic retention factors corrected with MW to the nth power. The best fit was obtained for $k_{(I A M)}$ with $n=2.5$. The same group continued their study combining IAM retention with hepatic metabolic clearance to model the oral absorption potential for a limited set of 15 drugs [54]. Yen et al. reported a reciprocal correlation between the negative value of the IAM retention factor $\left(-1 / k_{(\mathrm{IAM})}\right)$ and human intestinal absorption for 52 drugs $(R=0.64)$. This correlation was further improved upon the inclusion of molecular descriptors expressing molecular size and shape, solubility and polarity $(R=0.83)$ [55]. Kotecha et al. suggested that as intestinal absorption occurs along the gastrointestinal tract and mainly in the small intestine with a $\mathrm{pH}$ gradient, the maximum $\log k_{\mathrm{w}(\mathrm{IAM})}$, measured in a certain $\mathrm{pH}$ range (e.g. 4.5-7.4) and defined as $\log k_{\mathrm{w}(\mathrm{IAM})}^{\text {best }}$ should be considered [32,33]. The general model proposed by Kotecha et al. is a sigmoidal function of \%HOA, which can be improved by additional physicochemical parameters, leading to the general Eq. (10):

$$
\% \mathrm{HOA}=\frac{100}{1+10^{-\left(\alpha_{0}+\alpha_{1} \times x_{1}+\alpha_{2} \times x_{2}+\ldots+\alpha_{n} x_{n}\right)}}
$$

where $X_{1}$ stands for $\log k_{w(I A M)}^{\text {best }}, X_{2}, X_{3}, . . X_{n}$ are additional physicochemical properties and $\alpha_{0}, \alpha_{1}, \alpha_{2}, . ., \alpha_{n}$ are regression coefficients obtained by non-linear fitting. The best model for 27 structurally diverse drugs was obtained using $\log k_{\mathrm{w}(\mathrm{IAM})}^{\text {best }}$ in combination with the polar surface area [33]. Tsopelas et al. used an extended data set of 85 drugs to fit $\log k_{w(I A M)}^{\text {best }}$ in Eq. (10). They obtained successfully validated models by including MW, the Abraham's hydrogen-bond acidity parameter $(A)$, both with a negative sign, and the negatively charged molecular fraction with a positive sign. If zwitterions were excluded from the data set the model will include $\mathrm{MW}$ and positively charged molecular fraction $\left(\mathrm{F}^{+}\right)$, both with a negative sign. The negative sign of $\mathrm{F}^{+}$indicates that electrostatic interactions with the phosphate anions on the IAM surface are over expressed and may be associated with the binding component, reflecting drug-membrane interactions besides membrane permeability. Analogous models in terms of statistics and predictive performance were obtained by replacing the IAM retention factors with octanol-water distribution coefficients. The main difference between the two models relies on the opposite sign of the $\mathrm{F}^{+}$parameter (positive in the $\log D^{\text {best }}$ model, negative in the IAM model) attraction forces to the negatively charged inner membrane, which do play a role in absorption but are not expressed in the octanol-water system. In addition, the hydrogen bond acidity $\mathrm{A}$ is statistically non-significant in the $\log D^{\text {best }}$ model [17].

IAM retention factors have also served to model blood-brain barrier (BBB) penetration in combination with other physicochemical parameters such as molecular volume and structural indices. In an early study, the performance of IAM to model BBB penetration ( $\log B B$ ) was compared with octanol-water partitioning. The $\log \mathrm{BB} / \log k_{\mathrm{w}}$ correlations were slightly inferior in terms of statistics when compared to the corresponding $\log \mathrm{BB} / \log P$ data. Molecular volume was statistically significant with a negative contribution in both models [56]. Reichel and Begley found a significant correlation between the logarithm of brain uptake indices divided by the square root of the molecular weight (log BUI) of six biogenic amines with IAM retention $\left(R^{2}=0,747\right)$, although the same indices for six steroids correlated better with computational clogP than with $\log k_{\mathrm{w}(\mathrm{IAM})}\left(R^{2}=0.809\right.$ and $R^{2}=0.729$, respectively) [57]. Pehourcq et al. reported a parabolic relationship for the diffusion of cerebrospinal fluid (CSF) for a set of eight amyl propionate non-steroidal anti-inflammatory drugs. The inclusion of molecular weight led to an improved model [58]. Grumetto et al. established also parabolic relationships for the BBB passage for a set of 14 
basic drugs with retention on either IAM.PC.MG or IAM.PC.DD2 stationary phases. However, the better model obtained with $\log P$ directed the authors to use the $\Delta \log k_{\mathrm{w}(\mathrm{IAM})}$ parameter, assuming that the electrostatic interactions were encoded in the IAM retention as a factor that hinders drugs from transmembrane penetration [59]. They established significant linear negative log $\mathrm{BB} / \Delta \log k_{\mathrm{w}(\mathrm{IAM})}$, which were confirmed for an enlarged set of 40 structurally diverse drugs [25]. More recently, they have proposed a partial least square (PLS) model based on isocratic IAM retention factors in the presence of $30 \% \mathrm{MeOH}$ and in silico calculated physicochemical and topological indices. The PLS model constructed for 79 structurally unrelated analytes had however slightly inferior statistics to that obtained with retention factors on biopartitioning micellar chromatography in the presence of sodium dodecyl sulfate (SDS) [10]. Grumetto et al. [19] have found a parabolic relationship between $\log \mathrm{BB}$ and $\log k_{\mathrm{w}(\mathrm{IAM})}$ or $\Delta \log k_{\mathrm{w}(\mathrm{IAM})}$ measured on an IAMDD2 column, with an opposite sign for the linear term $\left(R^{2}=0.825\right.$ and $R^{2}=0.826$ respectively). Results were inferior when an IAM.MG column was used. Better parabolic relationships, however, were obtained with $\log P$ of the neutral form $\left(R^{2}=0.880\right)$ [59]. The same research group performed a comparative study between IAM retention indices ( $\log k_{\mathrm{w}(\mathrm{IAM})}$ and $\left.\Delta \log k_{\mathrm{w}(\mathrm{IAM})}\right)$ and PAMPA using a porcine brain lipid extract for the prediction of in situ BBB permeability where they found the $\Delta \log k_{\mathrm{w}(\mathrm{AM})}$ parameter to be more efficient than PAMPA [19].

Yoon et al. [60] developed a classification model based on IAM retention divided by MW to the $\mathrm{n}^{\text {th }}$ power $\left(k_{\mathrm{w}(\mathrm{IAM})} / \mathrm{MW}^{\mathrm{n}}\right)$. The best model was obtained using $k_{\mathrm{w}(\mathrm{IAM})} / \mathrm{MW}^{4}$ measured at $\mathrm{pH}$ values of 5.5 and 7.0 , with cut- off values equal to 1.01 and 0.85 , respectively.

Efforts have also been made for the employment of IAM chromatography as a tool to model the transdermal transport of drugs. In a comparative investigation, Lazaro et al. explored the potential of IAM and C18 stationary phases. They suggested that IAM can mimic skin partition but that skin permeation is closer to elution on reversed-phase columns [30]. Barbato et al. investigated a limited set of 12 drugs and used both the $\log k_{\mathrm{w}(\mathrm{IAM})}$ and the $\Delta \log k_{\mathrm{w}(\mathrm{IAM})}$ parameters with no correlation of skin permeability have been observed with $\log k_{\mathrm{w}(\mathrm{IAM})}$, but a negative correlation with $\Delta \log k_{\mathrm{w}(\mathrm{IAM})}$ was evident, indicating that polar and electrostatic interactions hinder molecules from crossing the skin layer, possibly due to repulsive forces [61].

A point of major importance lies in early drug safety assessment. Considering the binding component of the IAM retention mechanism, a high affinity to IAM stationary phases in the case of basic amphiphilic drugs may be considered as an indication of drug-induced phospholipidosis, a situation associated with excessive accumulation of phospholipids within lysosomes [62,63]. Investigations have shown good correlation between IAM-CHI indexes and phospholipidosis risk [64] or phospholipidogenic potential [65]. In terms of $\mathrm{CHI}_{(\mathrm{AM})}$ a value $>50$ may be related to phospholipidosis [41]. In a comparative study of 36 drugs, Jiang and Reilly developed a simple and rapid physicochemical screening approach based on chromatographic methods for predicting the potential of compounds to induce phospholipidosis [66]. They found an equally satisfactory correlation with the retention on electrokinetic chromatography based on docusate sodium salt $\left(R^{2}=0.87\right.$ and $R^{2}=0.86$ respectively) or with the logarithm of the volume of distribution $\log V_{\mathrm{d}}$ calculated from IAM chromatography (CHI-IAM) and human serum albumin chromatography, according to Eq. (11) as suggested by Hollosy et al [40].

$$
\begin{aligned}
& \log V_{d}=0.44 \cdot \log K_{(\text {IAM })}-0.22 \log K_{(\mathrm{HSA})}-0.66 \\
& \left(\mathrm{n}=179, \mathrm{R}^{2}=0.76, \mathrm{~s}=0.33\right)
\end{aligned}
$$

An analogous relationship, resulting from a combination of retention on both IAM and HSA stationary 
phases was reported by Valko et al. [67] to predict the unbound volume of distribution $\left(V_{\mathrm{du}}\right)$, defined as the volume of distribution divided by the unbound fraction (fu) of the drug in plasma: $V_{\mathrm{du}}=V_{\mathrm{d}} / \mathrm{fu}$.

$$
\begin{aligned}
& \log V_{\mathrm{du}}=0.43 \log K_{(\mathrm{IAM})}+0.23 \log K_{(\mathrm{HSA})}-0.72 \\
& \left(n=70, R^{2}=0.84, s=0.32\right)
\end{aligned}
$$

The unbound volume of distribution is an important pharmacokinetic parameter since it is in principle the reciprocal value of the maximum drug efficiency ( $D E_{\max }$ ) defined by Braggio et al. [68].

Cell accumulation and retention may be very important for drug action in particular for drugs which are intended to penetrate to intracellular targets or intracellular pathogens, as well as to maintain a prolonged presence in the inflammatory cells [69]. Encouraging results have been reported by Gordon et al. [70] when plotting the log cell concentration, determined by the High-Throughput Screening Assay RapidFire, versus $\log k_{(\mathrm{IAM})} \mathrm{A}$ positive trend was observed with a correlation coefficient $\mathrm{R}=0.69$, while the corresponding correlation with clogP showed a correlation coefficient $\mathrm{R}=0.42$.

\section{The use of IAM chromatography for the prediction of ecotoxicity}

The environment is continuously exposed to a great variety of chemical substances coming from industrial, agricultural, municipal and natural physicochemical and biological processes. New compounds are synthesized and tested as drugs, ingredients of cosmetics, food additives, pesticides, plasticizers or for general use in technical and industrial purposes, while others can be produced as a result of natural biological processes [71]. Responsible product design should take into consideration the possible risks in human health and to ecosystems. The evaluation of ecotoxicity indices is involved in the REACH regulation "no data no market" [72]. Among other eco-toxicological endpoints, median toxicity, i.e. lethal concentration $\mathrm{LC}_{50}$ in aquatic organisms and lethal dose, $\mathrm{LD}_{50}$, for terrestrial organisms as well as bioaccumulation are of major importance. Experimental measurements on biological organisms for the thousands of compounds for various uses are simply not possible.

In silico systems can be employed for ecotoxicological investigations, in which the logarithm of the octanol-water partition coefficient, symbolized in such studies as log $K_{\text {ow }}$, is a principal parameter [73]. Several authors have proposed linear solvation energy relationships (LSER) for ecological risk assessment [71,74-81]. However, failure in the selection of the appropriate model can result in a 1000-fold error in the estimated indices [82]. A popular predictive tool for environmental investigations is the estimation program interface (EPI Suite) software, freely available from the Environmental Protection Agency (EPA) [83]. Module BCFBAF, based on the work of Meylan et al. [84], calculates fish the bioconcentration factor and ECOSAR module, based on the work of Russon et al. [82], and estimates the aquatic toxicity of compounds.

Chromatographic techniques offer an experimental alternative for the rapid evaluation of ecotoxicological endpoints. Bio-partitioning liquid chromatography (BLC), as named by the Escuder-Gilabert group [85] was the first biomimetic liquid chromatographic approach implemented for ecotoxicological purposes. BLC uses micellar mobile phases formed mainly by the addition of the non-ionic surfactant polyoxyethylene(23)lauryl ether (Brij35). It has been employed for the predictions of bioconcentration factors [86,87], skin permeability [87] and toxicity ( $\mathrm{pLC}_{50}$ in fathead minnow) [88] of xenobiotics.

In recent years, IAM technology has unfolded new perspectives in the field of ecotoxicology. As IAM retention factors reflect permeability of xenobiotics through cell membranes governed by passive diffusion as well as membrane accumulation, it is plausible that it can be used to provide ecotoxicological indices to 
express median toxicity and bioconcentration or to construct a lipophilicity scale. Stepnowski and Storoniak [89] compared IAM and reversed phase retention as well as octanol-water partitioning as lipophilicity indices of hydrophilic imidazolium ionic liquids. The extrapolated $\log k_{\mathrm{w}(\mathrm{IAM})}$ values were higher than the corresponding $\log \mathrm{k}_{\mathrm{w}}$ on reversed-phase stationary phases and $\log P$, due to the development of electrostatic interactions of the positively charged nitrogen of the solutes with the phosphate anions of the IAM surface [89]. An analogous investigation was performed by Janicka [90], who compared IAM with reversed- phase, cholesterol phase and micellar chromatography. The authors used retention under the different chromatographic conditions as lipophilicity indices for 15 phenoxyacetic and carbamic acid derivatives with potential use as herbicides. Comparison with in silico calculated biological endpoints showed that all of the chromatographic indices were promising measures for the partitioning of chemicals in water- plant cuticle, water- human serum albumin and water- skin systems [90]. Cimpean et al. correlated IAM retention factors in the presence of $10 \%$ methanol with the non- specific toxicity (-log $\mathrm{LC}_{50}$ ) of the fathead minnow fish (Pimephales promelas) [91]. Hidalgo- Rodriguez et al. [92,93] and FernandezPumarega et al. [94] measured retention on several chromatographic and electrophoretic systems in order to find the best conditions to emulate non-specific aquatic toxicity and soil sorption. As aquatic toxicity endpoints, the lethal concentration $\left(\mathrm{LC}_{50}\right)$ of fathead minnow fish [92] and the tadpole narcosis concentration $\left(\log \left(\frac{1}{C_{n a r}}\right)\right.$ ) [94] were used, while soil sorption was also considered, expressed by the logarithm of the organic carbon normalized sorption coefficient, log $K_{\mathrm{oc}}$ [93]. Chromatographic and ecotoxicological data were submitted to LSER analysis. Comparison of the solvation parameter models (SPM) was based on the distance parameter $d$ (the difference between the coefficients of the respective SPMs), on the precision of biological-chromatographic correlations, on principal component analysis (PCA) of the coefficients of the compared systems and on dendrogram plots. Finally, the chromatographic indices selected according to the above tools were directly correlated with the ecotoxicological data. For aquatic toxicity, IAM chromatography along with micellar electrokinetic chromatography based on sodium taurocholate showed the best predictive performance [92,94]. Furthermore, IAM and electrokinetic chromatography based either on sodium dodecyl sulfate or sodium taurocholate provided the most precise correlation models for soil sorption. Especially, the IAM model showed the lowest experimental error as reflected in the standard deviation between the observed and calculated data [92].

The potential of IAM chromatography to predict bioconcentration of pharmaceutical compounds in aquatic organisms was recently investigated by our group [21]. Since very limited experimental bioconcentration factors (BCF) of pharmaceutical compounds are available, BCF values predicted by EPI Suite Software were used to compile a data set of 125 structurally diverse drugs. EPI Suite Software employs $\log k_{\text {ow }}$ (log $P$ calculated according to Meylan et al. [84]) as a major descriptor for BCF calculations, while for ionic and highly hydrophilic compounds an arbitrary value of log BCF $=0.50$ is assigned by the software. Therefore, such drugs were excluded from the analysis and were posteriori predicted by the IAM model. Highly significant linear log BCF/ log $k_{\mathrm{w}(\mathrm{IAM})}$ values were established. The constructed model was improved by the inclusion of the parameter BioWin5, which expresses the decrease in the bioaccumulation tendency as a result of the degradation potential of the compounds. BioWin5 is predicted by the EPI Suite Software and is included also in the EPI-BCF model. The log BCF/ log $k_{\text {w(IAM) }}$ relationship is shown in Eq.(13) [21]:

$$
\begin{aligned}
& \log \mathrm{BCF}=0.85 \cdot \log k_{\mathrm{w}(\mathrm{IAM})}^{7.4}-0.47 \cdot \operatorname{BioWin} 5+0.08 \\
& \left(n=77, R^{2}=0.761, s=0.437\right)
\end{aligned}
$$


Validation of the constructed models was based on a test set of drugs with experimentally determined $\mathrm{BCF}$ values reported in the literature. Predictions of the test set were compared with those provided by the EPI software and in most cases, they were in favour of the IAM model. This can be explained on the basis that $\log k_{\mathrm{w}(\mathrm{AM})}$ values involved electrostatic interactions due to ionization of the species at a pH close to those of a marine/ freshwater environment, while $\log k_{\text {ow }}$ is refers only to a neutral species expressing only a lipid- driven bioconcentration [95].

In recent work by Russo et al. [96], the IAM phospholipophilicity of seven bisphenol A analogues was compared with toxicity investigated on four different cell cultures. Results showed that the ranking of toxicity according to the cell cultures was partly consistent with the IAM retention, indicating that toxicity increases with increasing membrane affinity [96].

Unpublished results from our research group have revealed the ability of IAM chromatography to model median toxicity of a series of structurally diverse pesticides in aquatic organisms, involving fish species (e.g. Fathead Minnow and Rainbow Trout), Eastern Oyster and Water Flea as well as in honey bee. In comparison with octanol-water partitioning, models based on log $k_{\mathrm{w}(\mathrm{IAM})}$ demonstrate equal or superior statistics and, in some cases, they do not require the use of any additional physicochemical or topological parameters [97].

\section{Conclusions}

The nature of IAM retention is between passive diffusion and binding underlines its potential for numerous applications in the field of pharmaceutical and environmental sciences. An increasing number of publications suggest that IAM indices can be an equal or even a more effective tool than traditional octanol-water partitioning to express the passage of xenobiotics through biological membranes and barriers as well as drug-membrane interactions. The great advantages are speed, reproducibility and low analyte consumption, as well as the flexibility to provide more than one index (different isocratic $\log k_{\text {(IAM), }}$ extrapolated $\log k_{\mathrm{w}(\mathrm{IAM})}, \mathrm{CHI}_{(\mathrm{IAM})}$ or $\left.\Delta \log k_{\mathrm{w}(\mathrm{IAM})}\right)$ which can be alternatively used in correlation with biological endpoints. In addition, pharmacokinetic parameters like the volume of distribution or the fraction of unbound in tissue can be easily estimated on the basis of IAM chromatographic indices, while they may also serve for drug classification according to certain properties, such as CNS penetration or phospholipidosis potential. Less investigated has been the performance of IAM chromatography in predicting the toxicity of candidate drugs and ecotoxicity of xenobiotics as well as the combined use of IAM with other biomimetic chromatographic indices. Such studies need to be extended to further toxicity endpoints (e.g. cardiotoxicity) and ecotoxicity indices on terrestrial organisms (e.g. honey bee).

The promising results obtained mainly in modelling pharmacokinetic and drug efficacy related properties and, more recently, also in ecotoxicology suggest that a broader application of IAM chromatography in early drug discovery processes and in environmental science may save time and money in initial drug candidate selection and contribute to a reduced risk hazard of chemicals. The high intralaboratory reproducibility of $\log _{\mathrm{w}(\mathrm{IAM})}$ and $\mathrm{CH}_{(\mathrm{IAM})}$ values under analogous conditions permits their incorporation in to databases which could then serve as a tool for classification purposes, as well as to expand the drug- like concept, by incorporating limit values or ranges for IAM chromatographic indices. 


\section{Acknowledgements:}

supported by

ONASSIS FOUNDATION

Under the "Special Grant and Support Program for Scholars' Association Members" (Grant No. R ZN 004-1/2017-2018).

\section{References}

[1] K. Valko. Application of high- performance liquid chromatography based measurements of lipophilicity to model biological distribution. Journal of Chromatography A 1037 (2004) 299-310.

[2] K. Valko. Physicochemical and Biomimetic properties in drug discovery: Chromatographic techniques for lead optimization. Wiley, Hoboken, NJ, 2014.

[3] R. Kaliszan. Chromatography and capillary electrophoresis in modeling the basic process of drug action. TrAC- Trends in Analytical Chemistry 18 (1999) 400-410.

[4] J.G. Dorsey, M.G. Khaledi. Hydrophobicity estimations by reversed-phase liquid chromatography. Implications for biological partitioning processes. Journal of Chromatography A 656 (1993) 485-499.

[5] A. Leo, C. Hansch, D. Elkins. Partition coefficients and their uses. Chemical Reviews 71 (1971) 525616.

[6] M. Chrysanthakopoulos, F. Tsopelas, A. Tsantili- Kakoulidou. Biomimetic chromatography, a useful tool in the drug discovery process. In: E. Grunshka, N. Grinberg (Eds.). Advances in Chromatography, Vol. 51 (2014), pp. 91-125. New York, CRC Press.

[7] F. Tsopelas, T. Vallianatou, A. Tsantili- Kakoulidou. Advances in immobilized artificial membrane (IAM) chromatography for novel drug discovery. Expert Opinion on Drug Discovery 11 (2016) 473488.

[8] F. Tsopelas, C. Giaginis, A. Tsantili- Kakoulidou. Lipophilicity and biomimetic properties to support drug discovery. Expert Opinion in Drug Discovery 12 (2017) 885-896.

[9] M. Salary, M. Hadjmohammadi. Human serum albumin- mimetic chromatography based hexadecyltrimethylammonium bromide as a novel direct probe for protein binding of acidic drugs. Journal of Pharmaceutical and Biomedical Analysis 114 (2015) 1-7.

[10] G. Russo, L. Grumetto, R. Szucs, F. Barbato, F. Lynen. Determination of in vitro and in silico indexes for the modeling of blood-brain barrier partitioning of drugs via micellar and immobilized artificial membrane liquid chromatography. Journal of Medicinal Chemistry 60 (2017) 3739-3754.

[11] K. Valko. Lipophilicity and biomimetic properties measured by HPLC to support drug discovery. Journal of Pharmaceutical and Biomedical Analysis 130 (2016) 35-54.

[12] C.J. Van Leeuwen, J.L.M. Hermens, Risk assessment of chemicals. An introduction. Kluwer Academic, Dordrecht, 1995.

[13] V. Pliska, B. Testa, H. van de Waterbeemd (eds.), Lipophilicity in drug action and toxicology, VCH, Weinheim, 1996.

[14] Regies Technologies, Inc, 2016. Available at http://www.registech.com/ (retrieved at 8-5-2018).

[15] F. Tsopelas, A. Tsantili- Kakoulidou, M. Ochsenkühn- Petropoulou. Biomimetic chromatographic analysis of selenium species: Application for the estimation of their pharmacokinetic properties. Analytical and Bioanalytical Chemistry 397 (2010) 2171-2180.

[16] L. Grumetto, C. Carpentiero, P. Di Vaio, F. Frecentese, F. Barbato. Lipophilic and polar interaction forces between acidic drugs and membrane phospholipids encoded in IAM- HPLC indices: Their role in membrane partition and relationships with BBB permeation data. Journal of Pharmaceutical and Biomedical Analysis 75 (2013) 165-172. 
[17] F. Tsopelas, T. Vallianatou, A. Tsantili- Kakoulidou. The potential of immobilized artificial membrane chromatography to predict human oral absorption. European Journal of Pharmaceutical Sciences $\mathbf{8 1}$ (2016) 82-93.

[18] L. Grumetto, G. Russo, F. Barbato. Polar interactions drug/ phospholipids and comparison of their effectiveness in predicting drug human intestinal absorption. International Journal of Pharmacy $\mathbf{5 0 0}$ (2016) 275-290.

[19] L. Grumetto, G. Russo, F. Barbato. Immobilized artificial membrane HPLC derived parameters vs PAMPA- BBB data in estimating in situ measured blood-brain barrier permeation of drugs. Molecular Pharmaceutics 13 (2016) 2808-2816.

[20] F. Tsopelas, M. Tsagkrasouli, P. Poursanidis, M. Pitsaki, G. Vasios, P. Danias, I. Panderi, A. TsantiliKakoulidou. Retention behavior of flavonoids on immobilized artificial membrane chromatography and correlation with cell-based permeability. Biomedical Chromatography 32 (2018) e4108.

[21] F. Tsopelas, C. Stergiopoulos, L.A. Tsakanika, M. Ochsenkühn- Petropoulou, A. Tsantili- Kakoulidou. The use of immobilized artificial membrane chromatography to predict bioconcentration of pharmaceutical compounds. Ecotoxicology and Environmental Safety 139 (2017) 150-157.

[22] H. Liu, S. Ong, L. Glunz, C. Pidgeon. Predicting drug- membrane interactions by HPLC: Structural requirements of chromatographic surfaces. Analytical Chemistry 67 (1995) 355-3557.

[23] F. Barbato, G. Di Martino, L. Grumetto, M.I. La Rotonda. Can protonated $\beta$-blockers interact with biomembranes stronger than neutral isolipophilic compounds? A chromatographic study on three different phospholipid stationary phases (IAM- HPLC). European Journal of Pharmaceutical Sciences 25 (2005) 379-386.

[24] C. Giaginis, A. Tsantili- Kakoulidou. Alternative measures of lipophilicity: From octanol-water partitioning to IAM retention. Journal of Pharmaceutical Sciences 97 (2008) 2984-3004.

[25] L. Grumetto, G. Russo, F. Barbato. Indexes of polar interactions between ionizable drugs and membrane phospholipids measured by IAM- HPLC: Their relationships with data of blood-brain barrier passage. European Journal of Pharmaceutical Sciences 65 (2014) 139-146.

[26] F. Tsopelas, N. Malaki, T. Vallianatou, M. Chrysanthakopoulos, D. Vrakas, M. OchsenkühnPetropoulou, A. Tsantili-Kakoulidou. Insight into the retention mechanism on immobilized artificial membrane chromatography using two stationary phases. Journal of Chromatography A 1396 (2015) 25-33.

[27] G. Russo, L. Grumetto, F. Barbato, G. Vistoli, A. Pedretti. Prediction and mechanism elucidation of analyte retention on phospholipid stationary phases (IAM- HPLC) by in silico calculated physicochemical descriptors. European Journal of Pharmaceutical Sciences 99 (2017) 173-184.

[28] F. Tsopelas, M. Ochsenkühn- Petropoulou, A. Tsantili- Kakoulidou. Void volume markers in reversedphase and biomimetic liquid chromatography. Journal of Chromatography A 1217 (2010) 2847-2854.

[29] S. Ong, C. Pidgeon. Thermodynamics of solute partitioning into immobilized artificial membranes. Analytical Chemistry 67 (1995) 2119-2128.

[30] E. Lazaro, C. Rafols, M.H. Abraham, M. Roses. Chromatographic estimation of drug disposition properties by means of immobilized artificial membranes (IAM) and C18 columns. Journal of Medicinal Chemistry 49 (2006) 4861-4870.

[31] D. Vrakas, C. Giaginis, A. Tsantili- Kakoulidou. The different behaviour of structurally diverse basic and neutral drugs in the immobilized artificial membrane and reversed-phase high-performance liquid chromatography: Comparison with octanol-water partitioning. Journal of Chromatography $A$ 1116 (2006) 158-164.

[32] J. Kotecha, S. Shah, I. Rathod, G. Subbaiah. Relationships between immobilized artificial membrane chromatographic retention and human oral absorption of structurally diverse drugs. International Journal of Pharmaceutics 333 (2007) 127-135.

[33] J. Kotecha, S. Shah, I. Rathod, G. Subbaiah. Prediction of oral absorption in humans by experimental immobilized artificial membrane chromatography indices and physicochemical descriptors. International Journal of Pharmaceutics 360 (2008) 96-106. 
[34] D. Vrakas, C. Giaginis, A. Tsantili- Kakoulidou. Electrostatic interactions and ionization effect in immobilized artificial membrane retention. A comparative study with octanol-water partitioning. Journal of Chromatography A 1187 (2008) 67-78.

[35] F. Tsopelas, A. Tsantili- Kakoulidou, M. Ochsenkühn- Petropoulou. Lipophilicity, biomimetic retention profile and antioxidant activity of selenium species. Microchemical Journal 110 (2013) 711-718.

[36] Y. Marcus, Y. Migron. Polarity, hydrogen bonding, and structure of mixtures of water and cyanomethane. Journal of Physical Chemistry 95 (1991) 400-406.

[37] K. Morse, C. Pidgeon. Importance of mobile phase in immobilized artificial membrane chromatography. In: B. Testa, H. van de Waterbeemd, G. Folkers, R. Guy (Eds.), Pharmacokinetic optimization in drug research, Verlag Helvetica Chimica Acta, Zurich and Wiley- VCH, Weinheim, 2001, pp. 351-381.

[38] A. Taillardat- Bertschinger, P.A. Carrupt, F. Barbato, B. Testa. Immobilized artificial membrane HPLC in drug research. Journal of Medicinal Chemistry 46 (2003) 655-665.

[39] K. Valko, C.M. Du, C.D. Bevan, D.P. Reynolds, M.H. Abraham. Rapid- gradient HPLC method for measuring drug interactions with immobilized artificial membrane: comparison with other lipophilicity measures. Journal of Pharmaceutical Sciences 89 (2000) 1085-1096.

[40] F. Hollosy, K. Valko, A. Hersey, S. Nunhuck, G. Keri, C. Bevan. Estimation of volume of distribution in humans from high throughput HPLC- based measurements of human serum albumin binding and immobilized artificial membrane partitioning. Journal of Medicinal Chemistry 49 (2006) 6958-6971.

[41] K. Valko, S. Teague, C. Pidgeon. In vitro membrane binding and protein binding (IAM MB/PB technology) to estimate in vivo distribution: applications in early drug discovery. ADMET \& DMPK 5(1) (2017) 14-38.

[42] G.P. Van Balen, C.A.M. Martinet, G. Caron, G. Bouchard, M. Reist, P.A. Carrupt, R. Fruttero, A. Gasco, B. Testa. Liposome/ water lipophilicity: Methods, information content, and pharmaceutical applications. Medicinal Research Reviews 24 (2004) 299-324.

[43] J. Li, J. Sun, Z. He. Quantitative structure-retention relationship studies using immobilized artificial membrane chromatography I: amended linear solvation energy relationships with the introduction of a molecular electronic factor. Journal of Chromatography A 1132 (2006) 174-182.

[44] M.H. Abraham, W.E. Acree, A. Fahr, X. Liu. Analysis of immobilized artificial membrane retention factors for both neutral and ionic species. Journal of Chromatography A 1298 (2013) 44-49.

[45] G. Ermondi, M. Vallaro, G. Caron. Learning how to use IAM chromatography for predicting permeability. European Journal of Pharmaceutical Sciences 114 (2018) 385-390.

[46] F. Barbato. The use of immobilized artificial membrane (IAM) chromatography for determination of lipophilicity. Current Computer-Aided Drug Design 2 (2006) 341-352.

[47] S.T.J. Droge. Dealing with confounding pH-dependent surface charges in immobilized artificial HPLC columns. Analytical Chemistry 88 (2016) 960-967.

[48] M.R. Ledbetter, S. Gutsell, G. Hodges, S. O'Connor, J.C. Madden, P.H. Rowe, M.T.D. Cronin. Prediction of immobilized artificial membrane chromatography retention factors using theoretical molecular fragments and structural features. SAR and QSAR in Environmental Research 24 (2013) 661-678.

[49] B.H. Stewart, F.Y. Chung, B. Tait, C.J. Blankley, O.H. Chan. Hydrophobicity of HIV protease inhibitors by immobilized artificial membrane chromatography: application and significance to drug transport. Pharmaceutical Research 15 (1998) 1401-1406.

[50] T. Osterberg, M. Svensson, P. Lundahl. Chromatographic retention of drug molecules on immobilized liposomes prepared from egg phospholipids and from chemically pure phospholipids. European Journal of Pharmaceutical Sciences 12 (2001) 427-439.

[51] E.C. Chan, W.L. Tan, P.C. Ho, L.J. Fang. Modelling Caco-2 permeability of drugs using immobilized artificial membrane chromatography and physicochemical descriptors. Journal of Chromatography $A$ 1072 (2005) 159-168. 
[52] J. Keldenich. Measurement and prediction of oral absorption. Chemistry and Biodiversity 6 (2009) 2000-2013.

[53] C.H. Yoon, B.S. Shin, H.S. Chang, L.S. Kwon, H.Y. Kim, S.E. Yoo, S.D. Yoo. Rapid screening of drug absorption potential using the immobilized artificial membrane phosphatidylcholine column and molar volume. Chromatographia 60 (2004) 399-404.

[54] B.S. Shin, C.H. Yoon, J.P. Balthasar, B.Y. Choi, S.H. Hong, H.J. Kim, J.B. Lee, S.W. Hwang, S.D. Yoo. Prediction of drug bioavailability in humans using immobilized artificial membrane phosphatidylcholine column chromatography and in vitro hepatic metabolic clearance. Biomedical Chromatography 23 (2009) 764-769.

[55] T.E. Yen, S. Agatonovic- Kustrin, A.M. Evans, R.L. Nation, J. Ryand. Prediction of drug absorption based on immobilized artificial membrane (IAM) chromatography separation and calculated molecular descriptors. Journal of Pharmaceutical and Biomedical Analysis 38 (2005) 472-478.

[56] T. Salminen, A. Pulli, J. Taskinen. Relationship between immobilized artificial membrane chromatographic retention and the brain penetration of structurally diverse drugs. Journal of Pharmaceutical and Biomedical Analysis 15 (1997) 469-477.

[57] A. Reichel, D.J. Begley. Potential of immobilized artificial membranes for predicting drug penetration across the blood-brain barrier. Pharmaceutical Research 15 (1998) 1270-1274.

[58] F. Pehourcq, M. Matoga, B. Bannwarth. Diffusion of arylpropionate non- steroidal anti-inflammatory drugs into the cerebrospinal fluid: a quantitative structure-activity relationship approach. Fundamental and Clinical Pharmacology 18 (2004) 65-70.

[59] L. Grumetto, C. Carpentiero, F. Barbato. Lipophilic and electrostatic forces encoded in IAM-HPLC indexes of basic drugs: their role in membrane partition and their relationships with BBB passage data. European Journal of Pharmaceutical Sciences 45 (2012) 685-692.

[60] C.H. Yoon, S.J. Kim, B.S. Shin, K.C. Lee, S.D. Yo. Rapid screening of blood-brain barrier penetration of drugs using the immobilized artificial membrane phosphatidylcholine column chromatography. Journal of Biomolecular Screening 11 (2006) 13-20.

[61] F. Barbato, B. Cappello, A. Miro, M.I. La Rotonda, F. Quaglia. Chromatographic indexes on immobilized artificial membranes for the prediction of transdermal transport of drugs. Farmaco 53 (1998) 655-661.

[62] B.R. Berridge, L.A. Chatman, M. Odin, A.E. Schultze, P.E. Losco, J.T. Meehan, T. Peters, S.L. Vonderfecht. Phospholipidosis in nonclinical toxicity studies. Toxicologic Pathology 35 (2007) 325.

[63] U.M. Hanumegowda, G. Wenke, A. Regueiro- Ren, R. Yordanova, J.P. Corradi, S.P. Adams. Phospholipidosis as a function of basicity, lipophilicity and volume of distribution of compounds. Chemical Research in Toxicology 23 (2010) 749-755.

[64] J.M. Alakoskela, P. Vitovic, P.K.J. Kinnunen. Screening for the drug- phospholipid interaction: Correlation to phospholipidosis. ChemMedChem 4 (2009) 1224-1251.

[65] A. Casartelli, M. Bonato, P. Cristofori, F. Crivellente, G. Dal Negro, I. Masotto, C. Mutinelli, K. Valko, V. Bonfante. A cell-based approach for the early assessment of the phospholipidogenic potential in pharmaceutical research and drug development. Cell Biology and Toxicology 19 (2003) 161-176.

[66] Z. Jiang, J. Reilly. Chromatography approaches for early screening of the phospholipidosis- inducing potential of pharmaceuticals. Journal of Pharmaceutical and Biomedical Analysis 61 (2012) 184-190.

[67] K. Valko, S.B. Nunhuck, A.P. Hill. Estimating unbound volume of distribution and tissue binding by in vitro HPLC- based human serum albumin and immobilized artificial membrane- binding measurements. Journal of Pharmaceutical Sciences 100 (2011) 849-862.

[68] S. Braggio, D. Montanari, T. Rossi, E. Ratti. Application of drug efficiency index in drug discovery: a strategy towards low therapeutic dose. Expert Opinion in Drug Discovery 5 (2010) 609-618.

[69] G.W. Amsden. Advanced-generation macrolides: Tissue- directed antibiotics. International Journal of Antimicrobial Agents 18 (Supplement 1) (2001) 11-15. 
[70] L.J. Gordon, M. Allen, P. Artursson, M.M. Hann, B.J. Leavens, A. Mateus, S. Readshaw, K. Valko, G.J. Wayne, A. West . Direct Measurement of Intracellular Compound Concentration by RapidFire Mass Spectrometry Offers Insights into Cell Permeability. Journal of Biomolecular Screening 21 (2016) 156-164.

[71] K.R. Hoover, W.E. Acree, M.H. Abraham. Chemical toxicity correlations for several fish species based on the Abraham solvation parameter model. Chemical Research in Toxicology 18 (2005) 1497-1505.

[72] Regulation (EC) No 1907/ 2006 of the European Parliament and the Council of 18 December 2006 concerning the Registration, Evaluation, Authorisation and Restriction of Chemicals (REACH). Available at http://eur-lex.europa.eu/legal-content/EN/TXT/?uri=CELEX:32006R1907 (retrieved at 53-2018).

[73] European Commission, Joint Research Centre, European Union Reference Laboratory for alternatives to animal testing, 2018. Retrieved from https://eurl-ecvam.jrc.ec.europa.eu/validation-regulatoryacceptance/environmental-toxicity-fate/environmental-toxicity-bioaccumulation on 23-4-2018.

[74] M.H. Abraham, C. Rafols. Factors that influence tadpole narcosis. An LFER analysis. Journal of the Chemical Society, Perkin Transactions 210 (1995) 1843-1851.

[75] K.R.Bowen, K.B. Flanagan, W.E. Acree, M.H. Abraham, C. Rafols. Correlation of the toxicity of organic compounds to tadpoles using the Abraham model. The science of the Total Environment 371 (2006) 99-109.

[76] H. Laue, H. Gfeller, K.J. Jenner, J.W. Nichols, S. Kern, A. Natsch. Predicting the bioconcentration of fragrance ingredients by rainbow trout using measured rates of in vitro intrinsic clearance. Environmental Science and Technology 48 (2014) 9486-9495.

[77] N. Kluver, C. Vogs, R. Altenburger, B.I. Escher, S. Scholz. Development of a general baseline toxicity QSAR model for the fish embryo acute toxicity test. Chemosphere 164 (2016) 164-173.

[78] E. Perales, J.I. Garcia, E. Pires, L. Aldea, L. Lomba, B. Giner. Ecotoxicity and QSAR studies of glycerol ethers in Daphnia magna. Chemosphere 183 (2017) 277-285.

[79] S. Bakire, X. Yang, G. Ma, X. Wei, H. Yu, J. Chen, H. Lin. Developing predictive models for toxicity of organic chemicals to green algae based on mode of action. Chemosphere 190 (2018) 463-470.

[80] P. Gramatica, E. Papa, A. Sangion. QSAR modeling of cumulative environmental end- points for the prioritization of hazardous chemicals. Environmental Science: Processes and Impacts 20 (2018) 3847.

[81] J. He, T. Peng, X. Yang, H. Liu. Development of QSAR models for predicting the binding affinity of endocrine disrupting chemicals to eight fish estrogen receptor. Ecotoxicology and Environmental Safety 148 (2018) 211-219.

[82] C. L. Russom, S.P. Bradbury, S.J. Broderius, D.E. Hammermeister, R.A. Drummond. Predicting modes of toxic action from chemical structure: acute toxicity in the fathead minnow (Pimephales Promelas). Environmental Toxicology and Chemistry 16 (1997) 948-967.

[83] U.S., Environmental Protection Agency (EPA). Estimation Program Interface (EPI) Suite, Version 4.11., 2012. U.S. Environmental Protection Agency, Office of Pollution, Prevention and Toxics: Washington. Available at https://www.epa.gov/tsca-screening-tools/epi-suitetm-estimation-program-interface (retrieved at 21-4-2018).

[84] W.M. Meylan, P.H. Howard, R.S. Boethling, D. Aronson, H. Printu, S. Gouchie. Improved method for estimating bioconcentration/ bioaccumulation factor from octanol/ water partition coefficient. Environmental Toxicology and Chemistry 18 (1999) 664-672.

[85] L. Escuder- Gilabert, S. Sagrado, R.M. Villanueva- Camanas, M.J. Medina- Hernandez. Quantitative Retention- Structure- Activity relationship studies of local anesthetics by micellar liquid chromatography, Analytical Chemistry 70 (1998) 28-34.

[86] J.M. Bermudez- Saldana, L. Escuder- Gilabert, M.J. Medina- Hernandez, R.M. Villanueva- Camanas, S. Sagrado. Modelling bioconcentration of pesticides in fish using biopartitioning micellar chromatography. Journal of Chromatography A 1063 (2005) 153-160. 
[87] C-R. Yin, L-Y. Ma, J-G. Huang, L. Xu, Z-G. Shi. Fast profiling ecotoxicity and skin permeability of benzophenone ultraviolet filters using biopartitioning micellar chromatography based on penetrable silica spheres. Analytica Chimica Acta 804 (2013) 321-327.

[88] J.M. Bermudez- Saldana, L. Escuder- Gilabert, M.J. Medina- Hernandez, R.M. Villanueva- Camanas, S. Sagrado. Modelling bioconcentration of pesticides in fish using biopartitioning micellar chromatography. Journal of Chromatography B 852 (2007) 353-361.

[89] P. Stepnowski, P. Storoniak. Lipophilicity and metabolic route prediction of imidazolium ionic liquids. Environmental Science and Pollution Research 12 (2005) 199-204.

[90] M. Janicka. Correlations between chromatographic parameters and bioactivity predictors of potential herbicides. Journal of Chromatographic Science 52 (2014) 676-684.

[91] Cimpean D.M., Poole C.F. Systematic search for surrogate chromatographic models of biopartitioning processes. Analyst 127 (2002) 724-729.

[92] M. Hidalgo- Rodriguez, E. Fuguet, C. Rafols, M. Roses. Modelling nonspecific toxicity of organic compounds to the fathead minnow fish by means of chromatographic systems. Analytical Chemistry 84 (2012) 3446-3452.

[93] M. Hidalgo- Rodriguez, E. Fuguet, C. Rafols, M. Roses. Performance of chromatographic systems to model soil- water sorption. Journal of Chromatography A 1252 (2012) 136-145.

[94] A. Fernandez- Pumarega, S. Amezqueta, E. Fuguet, M. Roses. Tadpole toxicity prediction using chromatographic systems. Journal of Chromatography A 1418 (2015) 167-176.

[95] F. Grisoni, V. Consonni, S. Villa, M. Vighi, R. Todeschini. QSAR models for bioconcentration: Is the increase in the complexity justified by more accurate predictions? Chemosphere 127 (2015) 171-179.

[96] G. Russo, A. Capuozzo, F. Barbato, C. Irace, R. Santamaria, L. Grumetto. Cytotoxicity of seven bisphenol analogues compared to bisphenol $\mathrm{A}$ and relationships with membrane affinity data. Chemosphere 201 (2018) 432-440.

[97] C. Stergiopoulos, D. Makarouni, M. Ochsenkuhn- Petropoulou, A. Tsantili- Kakoulidou, F. Tsopelas. Immobilized artificial membrane chromatography as a tool for the prediction of ecotoxicity of pesticides. 2018. (unpublished results). 